\title{
Dissociation reactions of free tetrapyridinium tetracations and of their
}

catenanes

Jana Roithová, Petr Milko, Claire L. Ricketts, Detlef Schröder,* Thierry Besson, Václav Dekoj, and Martin Bělohradský

\section{SUPPLEMENTARY MATERIAL}

\section{Complete list of authors of reference 9.}

Amabilino, D. B.; Ashton, P. R.; Brown, C. L.; Córdova, E.; Godínez, L. A.; Goodnow, T. T.; Kaifer, A. E.; Newton, S. P., Pietraszkiewicz, M.; Philp, D.; Raymo, F. M.; Reder, A. S.; Rutland, M. T.; Slawin, A. M. Z.; Spencer, A. S.; Stoddart, J. F.; Williams, D. J. J. Am. Chem. Soc. 1995, 117,1271 , and references cited therein.

\section{Details of the synthesis of the tetrapyridinium cations 2 and 3}

Dicationic "horseshoe" X1. 1,4-bis(bromomethyl)triptycene (2 mmol, $880 \mathrm{mg}$ ) was dissolved in $25 \mathrm{~mL}$ acetonitrile and put into gas-tight syringe connected with linear doser. 4,4'-bipyridine (16 eq., $5.00 \mathrm{~g})$ was dissolved in $25 \mathrm{~mL}$ acetonitrile and put into oil bath $\left(85{ }^{\circ} \mathrm{C}\right)$ equipped with a magnetic stirrer. The solution of 1,4-bis(bromomethyl)triptycene was added dropwise within 4 hours into the refluxing solution of bipyridine under argon atmosphere, and the reaction mixture was further heated for 8 hours. It is recommended to protect reaction mixture against daylight by aluminium foil. Then reaction mixture was filtered and collected product was washed with $5 \mathrm{~mL}$ acetonitrile and $200 \mathrm{~mL}$ diethylether. The crude product was then dissolved in an acetone-water mixture (1:1), ammonium hexafluorophosphate $(2.6 \mathrm{~g})$ was added to the solution. Acetone was evaporated from the solution, after 24 hours white crystals were separated $(1.462 \mathrm{~g}$, yield 83\%, m.p. $=317-319^{\circ} \mathrm{C} / \mathrm{decomp} . /$ ).

EA: Calc.: $\mathrm{C}=57.14 \%, \mathrm{H}=3.65 \%, \mathrm{~N}=6.34 \%$, Found: $\mathrm{C}=57.02 \%, \mathrm{H}=3.94 \%, \mathrm{~N}=6.42 \%$.

${ }^{1} \mathbf{H}$ NMR (d $\mathrm{d}_{6}$-acetone): 6.26, s, $2 \mathrm{H}\left({ }^{\text {tert. }} \mathrm{C}-\mathrm{H}\right), 6.59, \mathrm{~s}, 4 \mathrm{H}\left(\mathrm{CH}_{2}\right) ; 6.99, \mathrm{~m}, 4 \mathrm{H}$ (arom.); 7.42, m, 4H (arom.); 7.46, s, 2H (arom.); 8.02, dd, 4H (BiPy arom.); 8.72, d (J=7.00 Hz), 4H (BiPy heteroarom.); 8.90, dd $(\mathrm{J} 1=1.69 \mathrm{~Hz}, \mathrm{~J} 2=4.51 \mathrm{~Hz}), 4 \mathrm{H}$ (BiPy heteroarom.); 9.31, d (J=6.99 Hz), 4H (BiPy heteroarom.).

Tetracationic cyclophane $\left[\mathbf{2}^{\mathbf{4 +}}\left(\mathbf{P F}_{\mathbf{6}}\right)_{\mathbf{4}}{ }^{-}\right]$. Template-directed synthesis was used successfully ${ }^{1}$ with 1,5-bis(methoxytetraethyleneglycolyl)naphtalene as a template. Dicationic compound X1 (283 mg, $0.32 \mathrm{mmol}$ ), 1,5-bis(methoxytetraethyleneglycolyl)naphtalene $(260 \mathrm{mg}, 0,48 \mathrm{mmol}$ ) and paraxylyl-dibromide $(85 \mathrm{mg}, 0.32 \mathrm{mmol})$ were dissolved in anhydrous acetonitrile $(10 \mathrm{~mL})$ and stirred under argon atmosphere for one week at room temperature. Acetonitrile was then evaporated from the violet reaction mixture and residue was purified by column chromatography on silicagel using a mixture of methanol $+2 \mathrm{M}$ aqueous solution of $\mathrm{NH}_{4} \mathrm{Cl}+$ nitromethane (ratio 7:2:1) as an eluent. Fractions containing product were partially evaporated, ammonium chloride was filtered off and washed with $20 \mathrm{ml}$ acetone. Ammonium hexafluorophosphate was added (300 mg) to the filtrate. Crystals of crude product were separated, dissolved in acetone-water mixture (1:1), ammonium hexafluorophosphate $(100 \mathrm{mg})$ was added to the solution. Acetone was then evaporated from the solution, after 24 hours white crystals were separated $\left(213 \mathrm{mg}\right.$, yield $52 \%$, m.p. $=327^{\circ} \mathrm{C}$, decomp. $)$. 

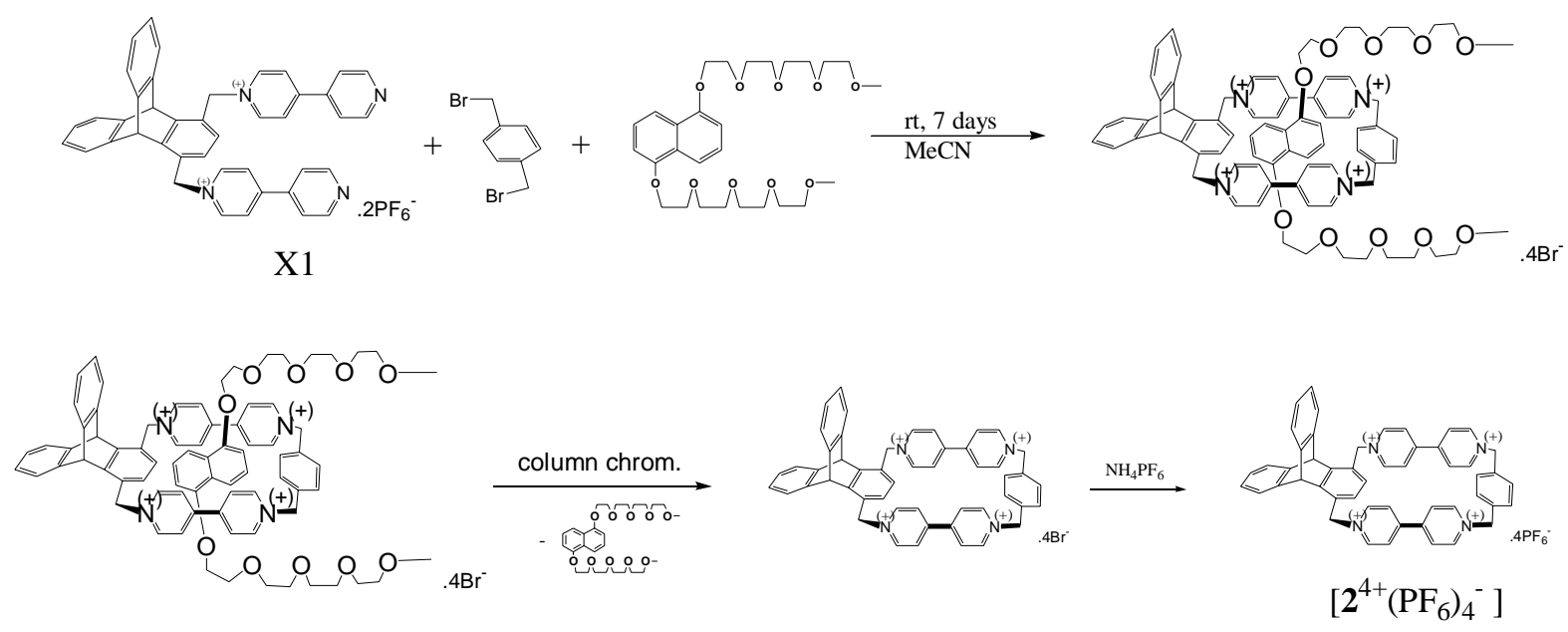

${ }^{1}$ H NMR, ${ }^{13}$ C NMR:

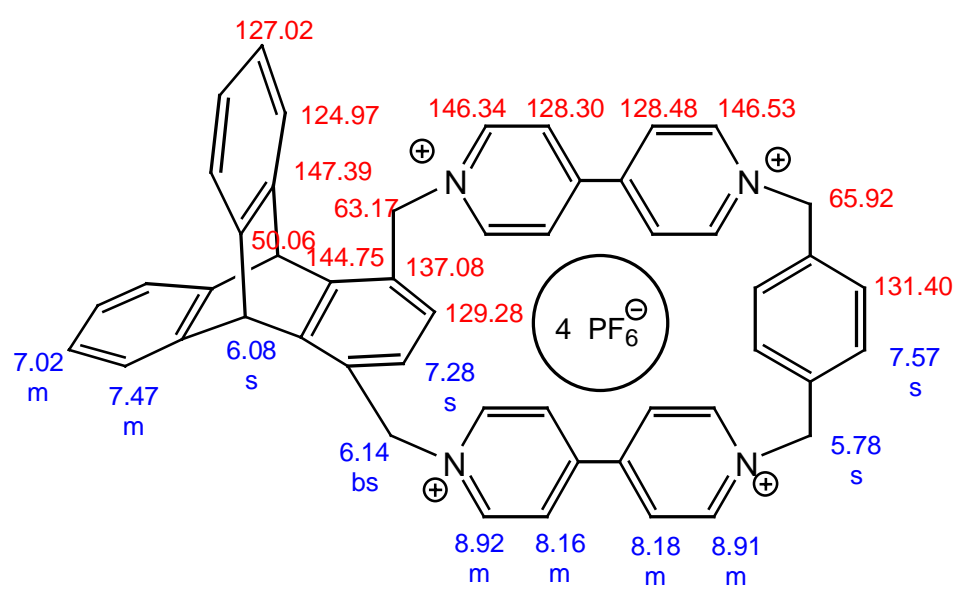

Tetracationic cyclophane $\left[3^{4+}\left(\mathrm{PF}_{6}\right)_{4}^{-}\right]$.

Template-directed synthesis was applied using 1,5-bis(methoxytetraethyleneglycolyl)naphtalene as a template. ${ }^{\mathrm{S} 1}$ The dicationic compound $\mathbf{X 1}(283 \mathrm{mg}, 0.32 \mathrm{mmol}), \quad 1,5-$ bis(methoxytetraethyleneglycolyl)naphtalene $\quad(260 \quad \mathrm{mg}, \quad 0,48 \quad \mathrm{mmol}), \quad$ and $1,4-$ bis(bromomethyl)triptycene $(141 \mathrm{mg}, 0.32 \mathrm{mmol})$ were dissolved in anhydrous acetonitrile $(10 \mathrm{~mL})$ and stirred for one week at room temperature under argon atmosphere. Acetonitrile was then evaporated from the violet reaction mixture, and the residue was purified by column chromatography on silicagel using a mixture of methanol $+2 \mathrm{M}$ aqueous solution of $\mathrm{NH}_{4} \mathrm{Cl}+$ nitromethane (ratio 7:2:1) as eluent. Fractions containing the product were partially evaporated, ammonium chloride was filtered off and washed with $20 \mathrm{ml}$ acetone. Ammonium hexafluorophosphate $(300 \mathrm{mg}$ ) was added to the filtrate. Crystals of crude product were separated, dissolved in acetone-water mixture (1:1), ammonium hexafluorophosphate (100 mg) was added to the solution. Acetone was then evaporated from the solution, after 24 hours white crystals were separated $\left(218 \mathrm{mg}\right.$, yield $47 \%$, m.p. $=360^{\circ} \mathrm{C}$, decomp. $)$ 


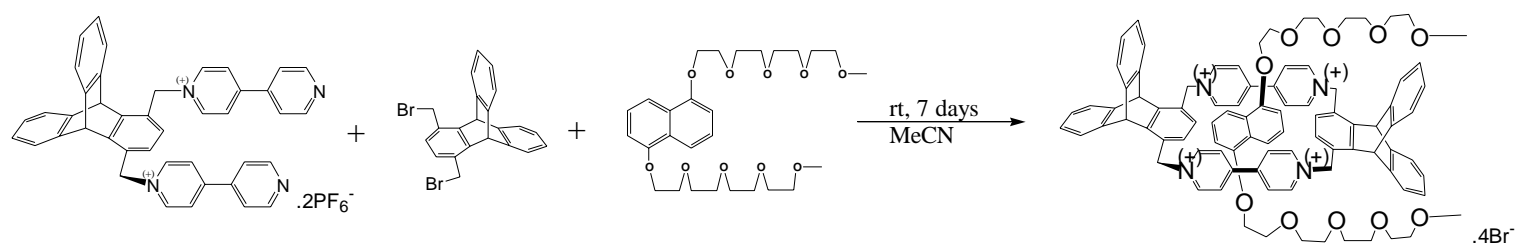

$\mathrm{X} 1$
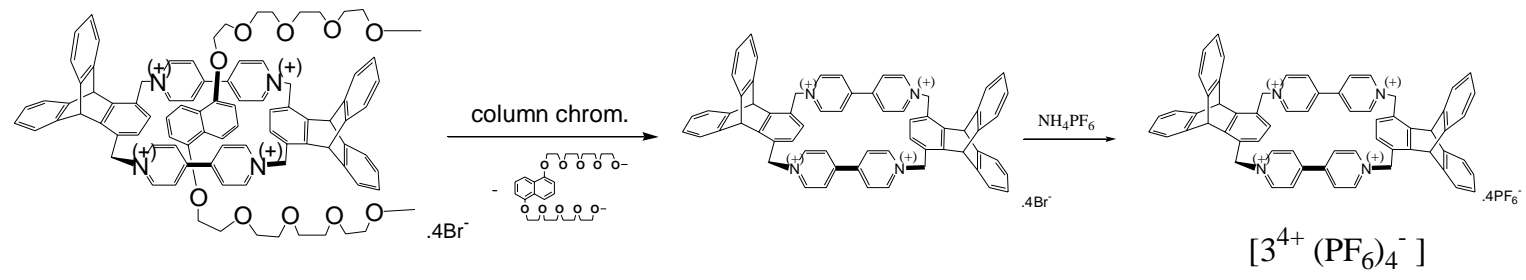

${ }^{1} \mathrm{H}$ NMR, ${ }^{13}$ C NMR:

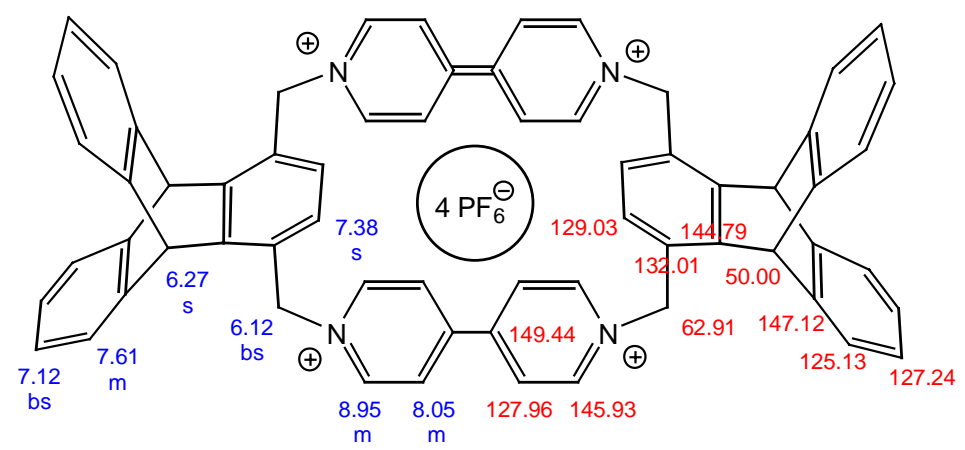

${ }^{1}$ H NMR and ${ }^{13} \mathrm{C}$ NMR study of Stoddart's tetracationic cyclophane ${ }^{\mathrm{S} 2}$ :

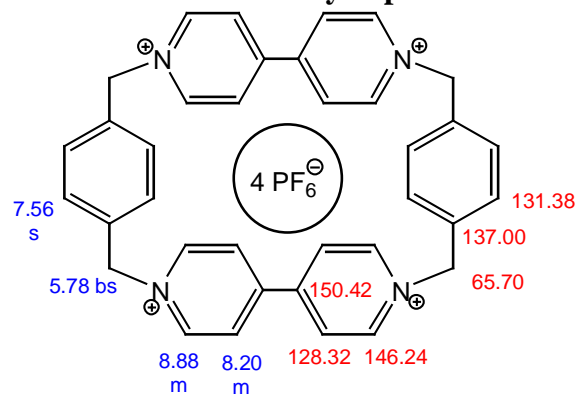

S1 Asakawa, M.; Dehaen, W.; L’abbe, G.: Menzer, S.; Nouwen, J.; Raymo, F. M.; Stoddart, J. F.; Williams, D. J. J.Org.Chem., 1996, 61, 9591-9595.

S2 Anelli, P. L.; Ashton, P. R.; Ballardini, R.; Balzani, V.; Delgado, M.; Gandolfi, M. T.; Goodnow, T. T.; Kaifer, A. E.; Philp, D.; Pietraszkewicz, M.; Prodi, L.; Reddington, M. V.; Slawin, A. M. Z.; Spencer, N.; Stoddart, J. F.; Vicent, C.; Williams, D. J. J. Am. Chem. Soc., 1992, 114, 193-218. 


\section{Cartesian coordinates of the computed structures discussed in the text with the same notation as used there.}

Tetracation $\mathbf{1}^{4+}$

State $=1-A \backslash H F=-1608.8315697$

NIMAG $=0$

Zero-point correction=

Thermal correction to Energy=

Thermal correction to Enthalpy=

0.604487 (Hartree/Particle)

0.635100

0.636044

Thermal correction to Gibbs Free Energy=

0.541735

Sum of electronic and zero-point Energies $=\quad-1608.227082$

Sum of electronic and thermal Energies $=\quad-1608.196470$

Sum of electronic and thermal Enthalpies $=\quad-1608.195526$

Sum of electronic and thermal Free Energies $=\quad-1608.289834$

Standard orientation:

\begin{tabular}{|c|c|c|c|c|c|}
\hline \multirow{2}{*}{$\begin{array}{l}\text { Center } \\
\text { Number }\end{array}$} & \multirow{2}{*}{$\begin{array}{l}\text { Atomic } \\
\text { Number }\end{array}$} & \multirow{2}{*}{\multicolumn{2}{|c|}{$\begin{array}{r}\text { Atomic } \\
\text { Type }\end{array}$}} & \multicolumn{2}{|c|}{ Coordinates (Angstroms } \\
\hline & & & & $\mathrm{X}$ & $\mathrm{Z}$ \\
\hline 1 & 6 & 0 & -4.634943 & -0.696039 & -1.212955 \\
\hline 2 & 6 & 0 & -4.956489 & -1.409697 & -0.047215 \\
\hline 3 & 6 & 0 & -5.320851 & -0.699475 & 1.102973 \\
\hline 4 & 6 & 0 & -5.320851 & 0.699475 & 1.102973 \\
\hline 5 & 6 & 0 & -4.956489 & 1.409696 & -0.047215 \\
\hline 6 & 6 & 0 & -4.634943 & 0.696039 & -1.212955 \\
\hline 7 & 6 & 0 & -4.919971 & -2.929145 & -0.039949 \\
\hline 8 & 7 & 0 & -3.487828 & -3.428999 & 0.009479 \\
\hline 9 & 6 & 0 & -2.895014 & -3.950462 & -1.089284 \\
\hline 10 & 6 & 0 & -1.531306 & -4.210517 & -1.110358 \\
\hline 11 & 6 & 0 & -0.745441 & -3.930161 & 0.019903 \\
\hline 12 & 6 & 0 & -1.415978 & -3.507731 & 1.182403 \\
\hline 13 & 6 & 0 & -2.776016 & -3.259833 & 1.148269 \\
\hline 14 & 6 & 0 & 0.745441 & -3.930161 & -0.019903 \\
\hline 15 & 6 & 0 & 1.531306 & -4.210515 & 1.110359 \\
\hline 16 & 6 & 0 & 2.895014 & -3.950461 & 1.089285 \\
\hline 17 & 7 & 0 & 3.487828 & -3.428999 & -0.009479 \\
\hline 18 & 6 & 0 & 2.776016 & -3.259835 & -1.148269 \\
\hline 19 & 6 & 0 & 1.415978 & -3.507733 & -1.182403 \\
\hline 20 & 6 & 0 & 4.919971 & -2.929145 & 0.039949 \\
\hline 21 & 6 & 0 & 4.956489 & -1.409696 & 0.047214 \\
\hline 22 & 6 & 0 & 5.320847 & -0.699475 & -1.102975 \\
\hline 23 & 6 & 0 & 5.320847 & 0.699475 & -1.102975 \\
\hline 24 & 6 & 0 & 4.956489 & 1.409697 & 0.047215 \\
\hline 25 & 6 & 0 & 4.634948 & 0.696039 & 1.212956 \\
\hline 26 & 6 & 0 & 4.634948 & -0.696039 & 1.212956 \\
\hline 27 & 6 & 0 & 4.919971 & 2.929145 & 0.039949 \\
\hline 28 & 7 & 0 & 3.487828 & 3.428999 & -0.009479 \\
\hline
\end{tabular}




$\begin{array}{rrrrrr}29 & 6 & 0 & 2.895013 & 3.950461 & 1.089285 \\ 30 & 6 & 0 & 1.531305 & 4.210515 & 1.110359 \\ 31 & 6 & 0 & 0.745441 & 3.930161 & -0.019903 \\ 32 & 6 & 0 & 1.415978 & 3.507733 & -1.182403 \\ 33 & 6 & 0 & 2.776016 & 3.259835 & -1.148269 \\ 34 & 6 & 0 & -0.745441 & 3.930161 & 0.019903 \\ 35 & 6 & 0 & -1.531306 & 4.210516 & -1.110358 \\ 36 & 6 & 0 & -2.895014 & 3.950462 & -1.089284 \\ 37 & 7 & 0 & -3.487828 & 3.428999 & 0.009479 \\ 38 & 6 & 0 & -2.776016 & 3.259833 & 1.148269 \\ 39 & 6 & 0 & -1.415979 & 3.507731 & 1.182403 \\ 40 & 6 & 0 & -4.919971 & 2.929145 & -0.039949 \\ 41 & 1 & 0 & -1.104602 & -4.624205 & -2.017719 \\ 42 & 1 & 0 & -3.528932 & -4.132366 & -1.949756 \\ 43 & 1 & 0 & -3.317683 & -2.885408 & 2.008534 \\ 44 & 1 & 0 & -0.885902 & -3.313619 & 2.107527 \\ 45 & 1 & 0 & 1.104602 & -4.624202 & 2.017720 \\ 46 & 1 & 0 & 3.528932 & -4.132363 & 1.949757 \\ 47 & 1 & 0 & 3.317683 & -2.885411 & -2.008535 \\ 48 & 1 & 0 & 0.885902 & -3.313622 & -2.107528 \\ 49 & 1 & 0 & 5.432845 & -3.347970 & -0.828187 \\ 50 & 1 & 0 & 5.368294 & -3.359131 & 0.937207 \\ 51 & 1 & 0 & 5.637806 & -1.225635 & -1.999424 \\ 52 & 1 & 0 & 5.637806 & 1.225635 & -1.999424 \\ 53 & 1 & 0 & 4.411328 & 1.221975 & 2.137253 \\ 54 & 1 & 0 & 4.411328 & -1.221975 & 2.137252 \\ 55 & 1 & 0 & 5.368294 & 3.359131 & 0.937208 \\ 56 & 1 & 0 & 5.432845 & 3.347970 & -0.828186 \\ 57 & 1 & 0 & 3.528932 & 4.132363 & 1.949758 \\ 58 & 1 & 0 & 1.104602 & 4.624202 & 2.017720 \\ 59 & 1 & 0 & 0.885902 & 3.313622 & -2.107528 \\ 60 & 1 & 0 & 3.317683 & 2.885411 & -2.008535 \\ 61 & 1 & 0 & -1.104602 & 4.624205 & -2.017719 \\ 62 & 1 & 0 & -3.528932 & 4.132366 & -1.949757 \\ 63 & 1 & 0 & -3.317683 & 2.885408 & 2.008534 \\ 64 & 1 & 0 & -0.885902 & 3.313619 & 2.107527 \\ 65 & 1 & 0 & -5.432846 & -3.347970 & 0.828186 \\ 66 & 1 & 0 & -5.368294 & -3.359131 & -0.937208 \\ 67 & 1 & 0 & -5.637813 & -1.225635 & 1.999421 \\ 68 & 1 & 0 & -5.637813 & 1.225635 & 1.999421 \\ 69 & 1 & 0 & -4.411320 & 1.221975 & -2.137251 \\ 70 & 1 & 0 & -4.411320 & -1.221975 & -2.137251 \\ 71 & 1 & 0 & -5.432846 & 3.347970 & 0.828186 \\ 72 & 1 & 0 & -5.368294 & 3.359131 & -0.937208\end{array}$


Tetracation $\mathbf{1}^{4+}$ (second conformer, slightly higher in energy)

State $=1-\mathrm{AlHF}=-1608.8305667$

$\mathrm{NIMAG}=0$

Zero-point correction $=$

Thermal correction to Energy=

Thermal correction to Enthalpy=

0.604679 (Hartree/Particle)

0.635210

0.636154

Thermal correction to Gibbs Free Energy $=\quad 0.542271$

Sum of electronic and zero-point Energies $=\quad-1608.225888$

Sum of electronic and thermal Energies $=\quad-1608.195357$

Sum of electronic and thermal Enthalpies $=\quad-1608.194413$

Sum of electronic and thermal Free Energies $=\quad-1608.288296$

Standard orientation:

\begin{tabular}{|c|c|c|c|c|c|}
\hline \multirow{2}{*}{$\begin{array}{l}\text { Center } \\
\text { Number }\end{array}$} & \multicolumn{2}{|c|}{ Atomic } & Atomic & \multicolumn{2}{|c|}{ Coordinates (Angstrom } \\
\hline & & Number & Type & $X$ & $\mathrm{Z}$ \\
\hline 1 & 6 & & -4.987036 & 0.788408 & 1.150767 \\
\hline 2 & 6 & & -4.963860 & 1.405955 & -0.107387 \\
\hline 3 & 6 & $\mathrm{C}$ & -4.991932 & 0.603027 & -1.256891 \\
\hline 4 & 6 & & -4.986993 & -0.788382 & -1.151059 \\
\hline 5 & 6 & & -4.963877 & -1.405929 & 0.107095 \\
\hline 6 & 6 & & -4.991991 & -0.603001 & 1.256598 \\
\hline 7 & 6 & $\mathrm{C}$ & -4.920056 & 2.920696 & -0.224629 \\
\hline 8 & 7 & & -3.4857 & 3.419975 & -0.197273 \\
\hline 9 & 6 & & -2.946 & 3.914751 & 1130 \\
\hline 10 & 6 & 5 & -1.586294 & 4.171727 & 1.035771 \\
\hline 11 & 6 & 5 & -0.743387 & 3.910846 & -0.058257 \\
\hline 12 & 6 & 5 & -1.355739 & 3.513197 & -1.260465 \\
\hline 13 & 6 & 5 & -2.717450 & 3.273423 & -1.301465 \\
\hline 14 & 6 & 5 & 0.743415 & 3.910843 & 0.058262 \\
\hline 15 & 6 & 5 & 1.586321 & 4.171714 & -1.035767 \\
\hline 16 & 6 & 5 & 2.946887 & 3.914731 & -0.941126 \\
\hline 17 & 7 & 7 & 3.485 & 3.419960 & 0.197279 \\
\hline 18 & 6 & 5 & 2.717476 & 3.273418 & 1.301472 \\
\hline 19 & 6 & 5 & 1.355765 & 3.513198 & 1.260473 \\
\hline 20 & 6 & 5 & 4.920077 & 2.920670 & 0.224635 \\
\hline 21 & 6 & 5 & 4.963871 & 1.405929 & 0.107389 \\
\hline 22 & 6 & 5 & 4.991929 & 0.602999 & 1.256892 \\
\hline 23 & 6 & 5 & 4.986980 & -0.788410 & 1.151058 \\
\hline 24 & 6 & 5 & 4.963867 & -1.405955 & -0.107098 \\
\hline 25 & 6 & 5 & 4.991994 & -0.603025 & -1.256599 \\
\hline 26 & 6 & 5 & 4.987049 & 0.788384 & -1.150765 \\
\hline 27 & 6 & 5 & 4.920069 & -2.920695 & -0.224345 \\
\hline 28 & 7 & 7 & 3.485801 & -3.419975 & -0.197073 \\
\hline 29 & 6 & 5 & 2.717527 & -3.273424 & -1.301310 \\
\hline 30 & 6 & 5 & 1.355814 & -3.513199 & -1.260392 \\
\hline 31 & 6 & 0 & 0.743391 & -3.910846 & -0.058219 \\
\hline
\end{tabular}




\begin{tabular}{rrrrrr}
32 & 6 & 0 & 1.586233 & -4.171726 & 1.035859 \\
33 & 6 & 0 & 2.946805 & -3.914749 & 0.941298 \\
34 & 6 & 0 & -0.743418 & -3.910844 & 0.058212 \\
35 & 6 & 0 & -1.355840 & -3.513204 & 1.260388 \\
36 & 6 & 0 & -2.717553 & -3.273423 & 1.301307 \\
37 & 7 & 0 & -3.485826 & -3.419960 & 0.197068 \\
38 & 6 & 0 & -2.946832 & -3.914727 & -0.941307 \\
39 & 6 & 0 & -1.586260 & -4.171710 & -1.035868 \\
40 & 6 & 0 & -4.920091 & -2.920670 & 0.224341 \\
41 & 1 & 0 & -1.206993 & 4.569163 & 1.970859 \\
42 & 1 & 0 & -3.622415 & 4.079528 & 1.772720 \\
43 & 1 & 0 & -3.215209 & 2.928335 & -2.199321 \\
44 & 1 & 0 & -0.781746 & 3.335357 & -2.162252 \\
45 & 1 & 0 & 1.207022 & 4.569147 & -1.970857 \\
46 & 1 & 0 & 3.622441 & 4.079499 & -1.772718 \\
47 & 1 & 0 & 3.215234 & 2.928334 & 2.199330 \\
48 & 1 & 0 & 0.781773 & 3.335367 & 2.162261 \\
49 & 1 & 0 & 5.356967 & 3.274147 & 1.160657 \\
50 & 1 & 0 & 5.438653 & 3.414623 & -0.598203 \\
51 & 1 & 0 & 5.056141 & 1.053688 & 2.243668 \\
52 & 1 & 0 & 5.037347 & -1.384677 & 2.057992 \\
53 & 1 & 0 & 5.056256 & -1.053714 & -2.243371 \\
54 & 1 & 0 & 5.037469 & 1.384651 & -2.057696 \\
55 & 1 & 0 & 5.357011 & -3.274176 & -1.160341 \\
56 & 1 & 0 & 5.438593 & -3.414651 & 0.598524 \\
57 & 1 & 0 & 3.215339 & -2.928338 & -2.199138 \\
58 & 1 & 0 & 0.781875 & -3.335359 & -2.162212 \\
59 & 1 & 0 & 1.206877 & -4.569162 & 1.970924 \\
60 & 1 & 0 & 3.622310 & -4.079525 & 1.772929 \\
61 & 1 & 0 & -0.781901 & -3.335375 & 2.162211 \\
62 & 1 & 0 & -3.215364 & -2.928343 & 2.199138 \\
63 & 1 & 0 & -3.622336 & -4.079491 & -1.772940 \\
64 & 1 & 0 & -1.206906 & -4.569140 & -1.970937 \\
65 & 1 & 0 & -5.356944 & 3.274178 & -1.160651 \\
66 & 1 & 0 & -5.438627 & 3.414651 & 0.598210 \\
67 & 1 & 0 & -5.056146 & 1.053719 & -2.243665 \\
68 & 1 & 0 & -5.037370 & -1.384647 & -2.057993 \\
69 & 1 & 0 & -5.056252 & -1.053691 & 2.243370 \\
70 & 1 & 0 & -5.037446 & 1.384674 & 2.057699 \\
71 & 1 & 0 & -5.438618 & -3.414621 & -0.598528 \\
72 & 1 & 0 & -5.357035 & -3.274149 & 1.160337 \\
--------------------------------------------------------- \\
\hline 5
\end{tabular}




$\begin{array}{lc}\text { Trication }\left[\mathbf{1}^{4+} \mathrm{PF}_{6}^{-}\right] & \\ \text {HF=-2549.8828247 } & 0.625237 \text { (Hartree/Particle) } \\ \text { NIMAG }=0 & 0.663922 \\ \text { Zero-point correction= } & 0.664867 \\ \text { Thermal correction to Energy= } & 0.553722 \\ \text { Thermal correction to Enthalpy= } & -2549.257588 \\ \text { Thermal correction to Gibbs Free Energy= } & -2549.218902 \\ \text { Sum of electronic and zero-point Energies= } & -2549.217958 \\ \text { Sum of electronic and thermal Energies= } & -2549.329103 \\ \text { Sum of electronic and thermal Enthalpies }= & \end{array}$

Standard orientation:

\begin{tabular}{|c|c|c|c|c|c|}
\hline \multirow{2}{*}{$\begin{array}{l}\text { Center } \\
\text { Number }\end{array}$} & \multirow{2}{*}{$\begin{array}{l}\text { Atomic } \\
\text { Number }\end{array}$} & \multirow{2}{*}{\multicolumn{2}{|c|}{$\begin{array}{l}\text { Atomic } \\
\text { Type }\end{array}$}} & \multicolumn{2}{|c|}{ Coordinates (Angstroms } \\
\hline & & & & $\mathrm{X}$ & $\mathrm{Z}$ \\
\hline 1 & 6 & 0 & -1.543840 & 4.351474 & 1.043823 \\
\hline 2 & 6 & 0 & -0.743766 & 3.945409 & -0.032426 \\
\hline 3 & 6 & 0 & -1.374174 & 3.372073 & -1.147287 \\
\hline 4 & 6 & 0 & -2.727612 & 3.106490 & -1.104088 \\
\hline 5 & 7 & 0 & -3.465351 & 3.427067 & -0.015075 \\
\hline 6 & 6 & 0 & -2.902201 & 4.080107 & 1.027282 \\
\hline 7 & 6 & 0 & 0.740712 & 3.945733 & 0.032262 \\
\hline 8 & 6 & 0 & 1.371387 & 3.372968 & 1.147271 \\
\hline 9 & 6 & 0 & 2.724978 & 3.108128 & 1.104219 \\
\hline 10 & 7 & 0 & 3.462597 & 3.428894 & 0.015171 \\
\hline 11 & 6 & 0 & 2.899128 & 4.081296 & -1.027400 \\
\hline 12 & 6 & 0 & 1.540620 & 4.351988 & -1.044042 \\
\hline 13 & 6 & 0 & 4.876439 & 2.925788 & -0.069368 \\
\hline 14 & 6 & 0 & 4.900478 & 1.406682 & -0.095842 \\
\hline 15 & 6 & 0 & 4.012581 & 0.695498 & -0.913566 \\
\hline 16 & 6 & 0 & 4.013214 & -0.693136 & -0.913729 \\
\hline 17 & 6 & 0 & 4.901717 & -1.403729 & -0.096153 \\
\hline 18 & 6 & 0 & 5.803659 & -0.697148 & 0.705956 \\
\hline 19 & 6 & 0 & 5.803051 & 0.700724 & 0.706105 \\
\hline 20 & 6 & 0 & 4.878833 & -2.922866 & -0.069914 \\
\hline 21 & 7 & 0 & 3.465373 & -3.427007 & 0.014883 \\
\hline 22 & 6 & 0 & 2.902142 & -4.079556 & -1.027717 \\
\hline 23 & 6 & 0 & 1.543768 & -4.350947 & -1.044256 \\
\hline 24 & 6 & 0 & 0.743748 & -3.945227 & 0.032171 \\
\hline 25 & 6 & 0 & 1.374259 & -3.372414 & 1.147253 \\
\hline 26 & 6 & 0 & 2.727711 & -3.106897 & 1.104108 \\
\hline 27 & 6 & 0 & -0.740740 & -3.945613 & -0.032454 \\
\hline 28 & 6 & 0 & -1.371465 & -3.372111 & -1.147033 \\
\hline 29 & 6 & 0 & -2.725036 & -3.107176 & -1.103704 \\
\hline 30 & 7 & 0 & -3.462599 & -3.428662 & -0.014854 \\
\hline 31 & 6 & 0 & -2.899090 & -4.081805 & 1.027240 \\
\hline 32 & 6 & 0 & -1.540594 & -4.352527 & 1.043651 \\
\hline
\end{tabular}




\begin{tabular}{|c|c|c|c|c|c|}
\hline 33 & 6 & 0 & -4.876502 & -2.925659 & 0.070137 \\
\hline 34 & 6 & 0 & -4.900468 & -1.406579 & 0.096175 \\
\hline 35 & 6 & 0 & -5.802340 & -0.700754 & -0.706652 \\
\hline 36 & 6 & 0 & -5.802901 & 0.697134 & -0.706780 \\
\hline 37 & 6 & 0 & -4.901620 & 1.403809 & 0.095948 \\
\hline 38 & 6 & 0 & -4.013707 & 0.693329 & 0.914312 \\
\hline 39 & 6 & 0 & -4.013108 & -0.695278 & 0.914406 \\
\hline 40 & 6 & 0 & -4.878843 & 2.922926 & 0.069736 \\
\hline 41 & 1 & 0 & -0.801439 & -3.022866 & -1.997893 \\
\hline 42 & 1 & 0 & -3.240837 & -2.584695 & -1.899409 \\
\hline 43 & 1 & 0 & -3.553027 & -4.345384 & 1.849810 \\
\hline 44 & 1 & 0 & -1.120560 & -4.849636 & 1.910808 \\
\hline 45 & 1 & 0 & 1.123913 & -4.847558 & -1.911786 \\
\hline 46 & 1 & 0 & 3.556158 & -4.342079 & -1.850561 \\
\hline 47 & 1 & 0 & 3.243394 & -2.585037 & 1.900312 \\
\hline 48 & 1 & 0 & 0.804183 & -3.024296 & 1.998555 \\
\hline 49 & 1 & 0 & 5.312762 & -3.360347 & -0.972259 \\
\hline 50 & 1 & 0 & 5.424720 & -3.320098 & 0.788839 \\
\hline 51 & 1 & 0 & 3.279590 & -1.206593 & -1.525884 \\
\hline 52 & 1 & 0 & 3.278465 & 1.208437 & -1.525563 \\
\hline 53 & 1 & 0 & 6.504497 & 1.230297 & 1.344406 \\
\hline 54 & 1 & 0 & 6.505554 & -1.226251 & 1.344154 \\
\hline 55 & 1 & 0 & 5.421811 & 3.323334 & 0.789569 \\
\hline 56 & 1 & 0 & 5.310283 & 3.363690 & -0.971553 \\
\hline 57 & 1 & 0 & 3.240812 & 2.586368 & 1.900379 \\
\hline 58 & 1 & 0 & 0.801356 & 3.024446 & 1.998435 \\
\hline 59 & 1 & 0 & 1.120582 & 4.848507 & -1.911542 \\
\hline 60 & 1 & 0 & 3.553101 & 4.344302 & -1.850125 \\
\hline 61 & 1 & 0 & -0.803967 & 3.023544 & -1.998328 \\
\hline 62 & 1 & 0 & -3.243188 & 2.584228 & -1.900086 \\
\hline 63 & 1 & 0 & -3.556275 & 4.342940 & 1.849985 \\
\hline 64 & 1 & 0 & -1.124103 & 4.848485 & 1.911149 \\
\hline 65 & 1 & 0 & -5.309876 & -3.363329 & 0.972656 \\
\hline 66 & 1 & 0 & -5.422256 & -3.323434 & -0.788443 \\
\hline 67 & 1 & 0 & -3.279454 & -1.208187 & 1.526995 \\
\hline 68 & 1 & 0 & -3.280534 & 1.206912 & 1.526901 \\
\hline 69 & 1 & 0 & -6.504308 & 1.226136 & -1.345599 \\
\hline 70 & 1 & 0 & -6.503344 & -1.230439 & -1.345350 \\
\hline 71 & 1 & 0 & -5.312770 & 3.360354 & 0.972107 \\
\hline 72 & 1 & 0 & -5.424747 & 3.320182 & -0.788992 \\
\hline 73 & 15 & 0 & -0.000053 & -0.000239 & 0.000017 \\
\hline 74 & 9 & 0 & 0.662391 & 1.162672 & -0.954457 \\
\hline 75 & 9 & 0 & -0.664061 & 1.161641 & 0.954598 \\
\hline 76 & 9 & 0 & -1.340649 & -0.001103 & -0.928490 \\
\hline 77 & 9 & 0 & 1.340528 & 0.000634 & 0.928502 \\
\hline 78 & 9 & 0 & 0.663975 & -1.162099 & -0.954558 \\
\hline 79 & 9 & 0 & -0.662447 & -1.163209 & 0.954328 \\
\hline
\end{tabular}




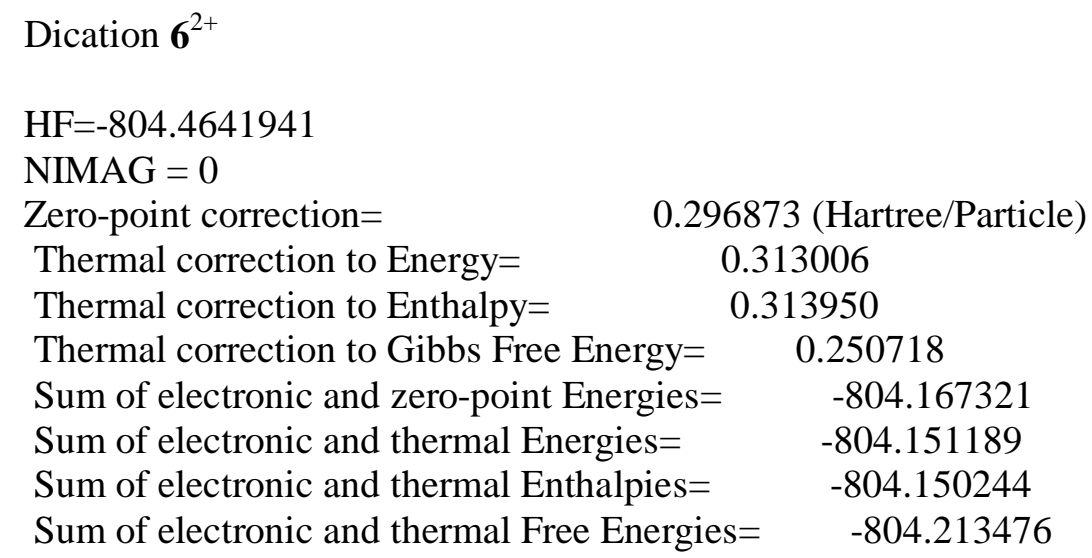

Standard orientation:

\begin{tabular}{|c|c|c|c|c|c|}
\hline \multirow{2}{*}{$\begin{array}{l}\text { Center } \\
\text { Number }\end{array}$} & \multirow{2}{*}{\multicolumn{2}{|c|}{$\begin{array}{l}\text { Atomic } \\
\text { Number }\end{array}$}} & \multirow{2}{*}{$\begin{array}{r}\text { Atomic } \\
\text { Type }\end{array}$} & \multicolumn{2}{|c|}{ Coordinates (Angstroms } \\
\hline & & & & $\mathrm{X}$ & $\mathrm{Z}$ \\
\hline 1 & 7 & 0 & -6.206446 & 0.906468 & -0.201576 \\
\hline 2 & 6 & 0 & -5.414568 & 1.360149 & 0.776643 \\
\hline 3 & 6 & 0 & -4.079548 & 0.984996 & 0.919601 \\
\hline 4 & 6 & 0 & -3.521957 & 0.097615 & -0.019789 \\
\hline 5 & 6 & 0 & -4.354621 & -0.372355 & -1.052461 \\
\hline 6 & 6 & 0 & -5.681482 & 0.054626 & -1.089597 \\
\hline 7 & 6 & 0 & -2.122088 & -0.325862 & 0.076913 \\
\hline 8 & 6 & 0 & -1.434727 & -0.359216 & 1.314139 \\
\hline 9 & 6 & 0 & -0.126961 & -0.778382 & 1.386198 \\
\hline 10 & 7 & 0 & 0.551671 & -1.166042 & 0.267897 \\
\hline 11 & 6 & 0 & -0.076258 & -1.146431 & -0.943530 \\
\hline 12 & 6 & 0 & -1.382801 & -0.734831 & -1.059676 \\
\hline 13 & 6 & 0 & 1.935513 & -1.667096 & 0.374336 \\
\hline 14 & 6 & 0 & 3.042430 & -0.655122 & 0.141538 \\
\hline 15 & 6 & 0 & 4.367200 & -1.157493 & 0.168963 \\
\hline 16 & 6 & 0 & 5.432738 & -0.309737 & -0.021858 \\
\hline 17 & 6 & 0 & 5.205319 & 1.093670 & -0.252032 \\
\hline 18 & 6 & 0 & 3.852207 & 1.582237 & -0.276881 \\
\hline 19 & 6 & 0 & 2.796305 & 0.717070 & -0.082830 \\
\hline 20 & 6 & 0 & 6.261887 & 1.953428 & -0.444328 \\
\hline 21 & 1 & 0 & 6.107585 & 3.015880 & -0.619465 \\
\hline 22 & 1 & 0 & 7.291372 & 1.602242 & -0.428252 \\
\hline 23 & 1 & 0 & 3.672923 & 2.639393 & -0.449211 \\
\hline 24 & 1 & 0 & 1.781271 & 1.096626 & -0.103092 \\
\hline 25 & 1 & 0 & 6.450131 & -0.689030 & -0.002083 \\
\hline 26 & 1 & 0 & 4.541685 & -2.215488 & 0.341165 \\
\hline 27 & 1 & 0 & 2.050906 & -2.496038 & -0.332170 \\
\hline 28 & 1 & 0 & 2.059715 & -2.103915 & 1.371410 \\
\hline 29 & 1 & 0 & 0.506333 & -1.473309 & -1.797014 \\
\hline 30 & 1 & 0 & 0.409356 & -0.841353 & 2.325879 \\
\hline 31 & 1 & 0 & -1.937873 & -0.096180 & 2.236546 \\
\hline 32 & 1 & 0 & -1.828311 & -0.720631 & -2.046811 \\
\hline
\end{tabular}




$\begin{array}{rrrrrr}33 & 1 & 0 & -4.006899 & -1.089527 & -1.788342 \\ 34 & 1 & 0 & -6.350684 & -0.306254 & -1.866227 \\ 35 & 1 & 0 & -5.865745 & 2.056204 & 1.478950 \\ 36 & 1 & 0 & -3.489848 & 1.414792 & 1.722369\end{array}$

